\title{
Effects of magnetic nanoparticles with different surface coating on the phase transitions of octylcyanobiphenyl liquid crystal
}

\author{
George Cordoyiannis, ${ }^{1}$ Lynn K. Kurihara, ${ }^{2}$ Luz J. Martinez-Miranda, ${ }^{3}$ Christ Glorieux, ${ }^{1}$ and Jan Thoen ${ }^{1, *}$ \\ ${ }^{1}$ Laboratorium voor Akoestiek en Thermische Fysica, Departement Natuurkunde en Sterrenkunde, Katholieke Universiteit Leuven, \\ Celestijnenlaan 200D, B-3001 Leuven, Belgium \\ ${ }^{2}$ Naval Research Laboratory, Washington, D.C. 20375, USA \\ ${ }^{3}$ Department of Materials Science and Engineering, University of Maryland, College Park, Maryland 200742-2115, USA
}

(Received 24 June 2008; published 7 January 2009)

\begin{abstract}
The impact of magnetic nanoparticles with different surface coating upon the isotropic-to-nematic and nematic-to-smectic- $A$ phase transitions of the liquid crystal octylcyanobiphenyl is explored by means of highresolution adiabatic scanning calorimetry. A shrinkage of the nematic range is observed, which is strongly dependent on the surface coating of the nanoparticles. The isotropic-to-nematic transition remains weakly first order while the nematic-to-smectic- $A$ is continuous with the effective critical exponent $\alpha$ values ( 0.35 and 0.39 , depending on the coating) between the pure octylcyanobiphenyl value of $0.31 \pm 0.03$ and the theoretical tricritical value of 0.5 .
\end{abstract}

DOI: 10.1103/PhysRevE.79.011702

PACS number(s): 64.70.M-, 47.65.Cb

\section{INTRODUCTION}

Liquid crystals (LCs) are soft materials that exhibit various mesophases and phase transitions between them and they are sensitive to perturbations. Consequently, systems of LCs in porous media appear as ideal candidates for the study of the quenched random disorder effects upon phase transitions and critical phenomena [1,2], and they have been studied extensively in the last 20 years. Aerogels [3,4], hydrophilic or hydrophobic silica aerosil networks [5-12], controlled porous glasses [13,14], vycor glasses [15], and anopores [16] were frequently chosen as the agents of disorder. In addition, dispersions of colloids [17], golden [18], ferroelectric [19], and magnetic nanoparticles $[20,21]$ in LCs have been studied particularly to enhance the optical, electrical, and magnetic properties and response. Several of these systems exhibit a considerable potential for applications [17,18,22,23].

The idea of ferrofluids composed from LCs doped with ferromagnetic grains appeared many years ago [24], aiming to enhance the coupling between LC order and external magnetic fields. Already in the 1970s some ferrofluids were successfully composed $[25,26]$. Nowadays, names such as ferronematics, ferrosmectics, or ferrohexagonals stand for nematic, smectic, or hexagonal LCs, respectively, doped with magnetic nanoparticles. They have been studied in both lyotropic and thermotropic LCs $[20,21,27,28]$, with the size and concentration effects being the main inquiry. The role of the surface coating has not been adequately explored so far [29]. In this work the effect of spherical magnetic nanoparticles with different surface coatings on the phase transitions from the isotropic-to-nematic $(I-N)$ and nematic-to-smectic $A$ $(N-\mathrm{Sm}-A)$ phase of the liquid crystal compound octylcyanobiphenyl (8CB) is explored by means of high-resolution adiabatic scanning calorimetry (ASC).

\footnotetext{
*jan.thoen@fys.kuleuven.be
}

\section{MATERIALS AND METHODS}

Spherical magnetic nanoparticles were used for this study, with a core diameter of $2 \mathrm{~nm}$. In order to probe the role of the surface chemistry, two mixtures of $8 \mathrm{CB}$ and magnetic nanoparticles with identical concentration $(30.6 \mathrm{wt} \%)$ and different coatings were chosen. In the first case, the nanoparticles were coated with aminopropyltriethoxysilane (APTS) and in the second case with mercaptohexadecanoic acid (MHDA) agents. Henceforth we will refer to the former as T1 and to the latter as T2. In Fig. 1 the chemical formulas of the surface agents are given. The APTS binds on the nanoparticles core through the silane, while MHDA binds through the sulfur group. Further details about the preparation of the samples can be found elsewhere [29,30].

The ASC measurements were performed in a computercontrolled calorimeter consisting of four stages. For the current measurements the inner stage was a $22 \mathrm{~g}$ tantalum cell, which contained $0.8 \mathrm{~g}$ of sample. The space between the cell and the three surrounding shields is vacuum-pumped in order to achieve excellent thermal insulation between them. ASC yields both the heat capacity $\left(C_{p}\right)$ and the enthalpy $(H)$ temperature dependence. It can efficiently distinguish between first-order and second-order phase transitions. The extremely slow scanning rates, that can be achieved in ASC, reveal subtle features of the specific heat capacity temperature profile $C_{p}(T)$ and allow the reliable determination of critical exponents. A detailed description of our apparatus can be found elsewhere [31,32].

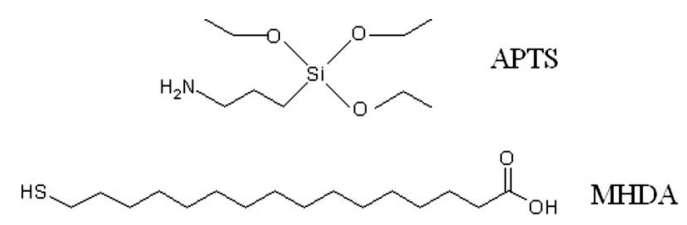

FIG. 1. The chemical formulas of the APTS and MHDA agents for the surface coating of the magnetic nanoparticles used in the samples $\mathrm{T} 1$ and $\mathrm{T} 2$, respectively. 
TABLE I. The features of all the ASC scans performed for the samples $\mathrm{T} 1$ and $\mathrm{T} 2$.

\begin{tabular}{lcccc}
\hline \hline Sample & Type of run & $\begin{array}{c}T \text { range } \\
(\mathrm{K})\end{array}$ & $\begin{array}{c}\text { Rate } \\
(\mathrm{mK} / \mathrm{min})\end{array}$ & Stirring \\
\hline $\mathrm{T} 1$ & Cooling & $319.15-299.15$ & 4.3 & Yes \\
$\mathrm{T} 1$ & Cooling & $319.15-299.15$ & 4.3 & No \\
$\mathrm{T} 1$ & Cooling & $310.15-300.15$ & 2.7 & Yes \\
$\mathrm{T} 2$ & Cooling & $319.15-299.15$ & 4.4 & Yes \\
$\mathrm{T} 2$ & Cooling & $319.15-299.15$ & 4.4 & No \\
\hline \hline
\end{tabular}

The samples were sonicated for a few hours at temperatures in the $N$ and then in the $I$ phase, before loading to the cell. Prior to the measurements a small metal stirring ball (from nonmagnetic stainless steel) was inserted in the cell together with the sample. During the measurements, by periodically changing the inclination of the whole apparatus (via an automatized mechanism), the stirring ball was moving back and forth inside the cylindrical cell in order to maintain the maximum possible homogeneity of the samples. For both samples sequential measurements were performed with and without mixing, in order to retrace possible differences in the thermodynamic behavior (e.g., due to phase separation).

\section{RESULTS AND DISCUSSION}

In this section the results for both kinds of nanoparticles using ASC are reported. The data were collected upon cooling the samples from the $I$ down to $\mathrm{Sm}-A$ phase. The main features of all the ASC runs can be found in Table I. It should be noted that the quoted rates are average values away from the transition; near the transition they are much slower.

It has been found that the $8 \mathrm{CB}$ exhibits a weakly first order $I-N$ transition at $313.95 \mathrm{~K}$ with a latent heat of $2.14 \pm 0.02 \mathrm{~J} / \mathrm{g}$, and a continuous $N-\mathrm{Sm}-A$ transition at

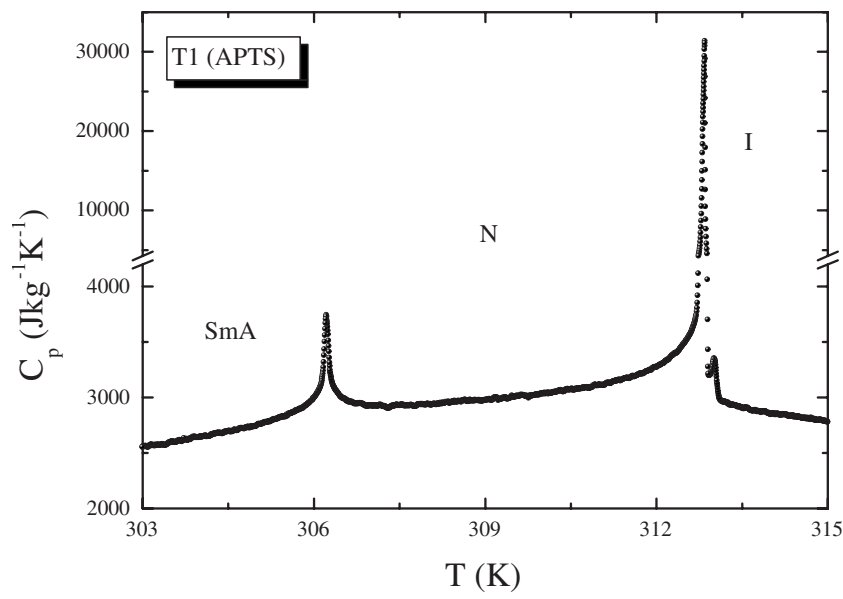

FIG. 2. The specific heat capacity versus temperature profile for the sample T1. Breakpoints and different scale below and above them are used along the $Y$ axis for more efficient representation of the curve at the $I-N$ transition.

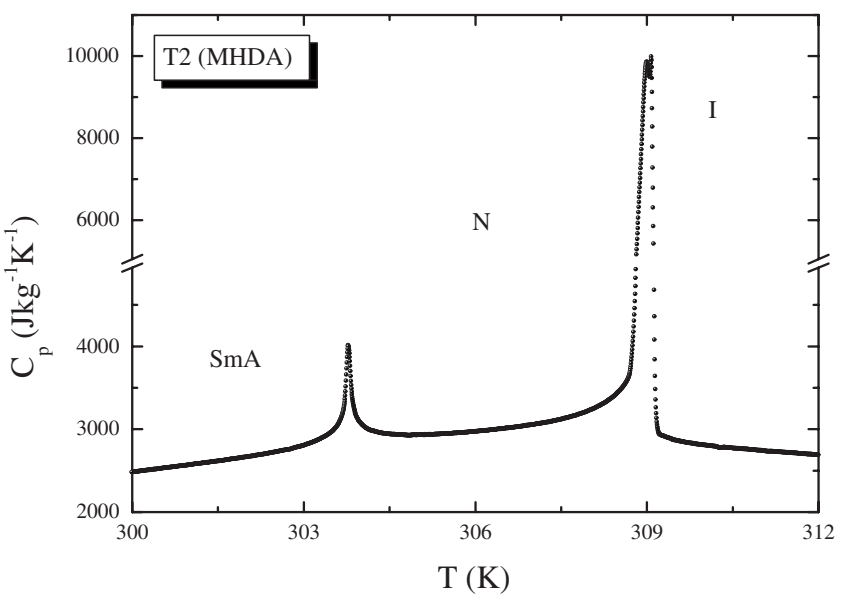

FIG. 3. The specific heat capacity versus temperature profile for the sample T2. Breakpoints and different scale below and above them are used along the $Y$ axis for more efficient representation of the curve at the $I-N$ transition.

$306.92 \mathrm{~K}$ with an effective critical exponent $\alpha=0.31 \pm 0.03$ [31]. These results were fully consistent with the anisotropic correlation lengths derived from x-ray measurements [33] within the frame of a hyperscaling relation [34].

In Fig. 2 the $C_{p}(T)$ curve is shown for the cooling run of the T1 sample, with stirring. The respective data are shown for the T2 sample in Fig. 3. No essential difference was found between runs with stirring and without stirring for any of the samples. A summary of the findings concerning the thermal behavior of $\mathrm{T} 1$ and $\mathrm{T} 2$, compared to pure $8 \mathrm{CB}$, is given in Table II.

The $I-N$ phase transition remains weakly first order, with pretransitional effects for both samples. The latent heat is $L$ $=1.55 \pm 0.03 \mathrm{~J} / \mathrm{g}$ for $\mathrm{T} 1$ and $L=1.48 \pm 0.04 \mathrm{~J} / \mathrm{g}$ for $\mathrm{T} 2$, which is smaller than the pure $8 \mathrm{CB}$ value $L$ $=2.14 \pm 0.02 \mathrm{~J} / \mathrm{g}$ [31]. The narrow $10 \pm 1 \mathrm{mK}$ coexistence range of $I$ and $N$ phases for bulk $8 \mathrm{CB}$ increases substantially here, being $110 \pm 10 \mathrm{mK}$ and $430 \pm 10 \mathrm{mK}$ for $\mathrm{T} 1$ and for $\mathrm{T} 2$, respectively. The $N-\mathrm{Sm}-A$ phase transition remains continuous for both samples and a detailed analysis is performed below in order to derive the critical exponents $\alpha_{\text {eff }}$. The nematic range decreases slightly in the case of $\mathrm{T} 1$ and fairly in the case of T2 (see Table II). For runs without stirring the

TABLE II. The transition temperatures, the latent heat of the $I-N$ transition, the nematic range, and the derived effective critical exponent $\alpha_{\text {eff }}$ for T1 and T2 samples (of this work) in comparison to pure 8CB (taken from Ref. [31]).

\begin{tabular}{lcccc}
\hline \hline Sample & $\begin{array}{c}T_{I N}(\mathrm{~K}) \\
{\left[L_{I N}(\mathrm{~J} / \mathrm{g})\right]}\end{array}$ & $\begin{array}{c}T_{N A} \\
(\mathrm{~K})\end{array}$ & $\begin{array}{c}N \text { range } \\
(\mathrm{K})\end{array}$ & $\alpha_{\text {eff }}$ \\
\hline $8 \mathrm{CB}$ & 313.95 & 306.92 & 7.03 & $0.31 \pm 0.03$ \\
& {$[2.14 \pm 0.02]$} & & & \\
$\mathrm{T} 1$ & 312.85 & 306.22 & 6.63 & $0.35 \pm 0.01$ \\
& {$[1.55 \pm 0.03]$} & & & \\
$\mathrm{T} 2$ & 309.05 & 303.78 & 5.27 & $0.39 \pm 0.01$ \\
& {$[1.48 \pm 0.04]$} & & & \\
\hline
\end{tabular}


transition temperatures were faintly shifted to lower values (in the order of few $\mathrm{mK}$ ) and only in the case of T2 the $I-N$ transition appeared less steep and with a wider phase coexistence range of $680 \pm 20 \mathrm{mK}$.

In the vicinity of a critical phase transition, the specific heat capacity has the following form:

$$
C_{p}=A^{ \pm}|t|^{-\alpha}+B
$$

with $A^{ \pm}$being the critical amplitudes above and below the transition temperature $T_{c}, t=\left(T-T_{c}\right) / T_{c}$ (reduced temperature), and $B$ the background term. Provided that both $C_{p}(T)$ and $H(T)$ are obtained from the same ASC run, one may define the quantity $C=\left(H-H_{c}\right) /\left(T-T_{c}\right)$ with $H$ and $H_{c}$ being the enthalpy at any temperature $T$ and at the critical transition temperature $T_{c}$, respectively. After a simple algebraic procedure one obtains [31]

$$
C-C_{p}=\frac{A^{ \pm}}{1-\alpha}|t|^{-\alpha},
$$

and in a $\log _{10}-\log _{10}$ plot of the quantities $C-C_{p}$ and $t$, the critical exponent can be determined from the slope $(-\alpha)$ of a linear fit. By using this method the background term $B$ is skillfully left out from the fitting procedure. Both wings of the $N-\mathrm{Sm}-A$ transition were fitted and the results are identical to the quoted values of $\alpha_{e f f}$. The data of the second slower cooling run were fitted for T1 (scanning rate of $2.7 \mathrm{mK} / \mathrm{min}$, with stirring) and the data of the first cooling for T2 (scanning rate of $4.4 \mathrm{mK} / \mathrm{min}$, with stirring).

As it can be seen in Fig. 4, the effective critical exponent $\alpha_{e f f}$ evolves from the value of $0.31 \pm 0.03$ of pure $8 \mathrm{CB}$ [31] to higher values, which are $0.35 \pm 0.01$ and $0.39 \pm 0.01$ for $\mathrm{T} 1$ and $\mathrm{T} 2$, respectively. It exhibits a trend from the anisotropic criticality of pure $8 \mathrm{CB}$ towards tricriticality. This is an important finding, since it shows a significant difference between the dispersions of the magnetic nanoparticles and the silica aerosil nanoparticles in liquid crystals. For the $N-\mathrm{Sm}-A$ transition of the latter, the effective critical exponents shift towards the three-dimensional- $X Y$ universality class when increasing the aerosil density [5,7,35-37], whereas the transition is smeared out for very high aerosil densities, deep in the so-called stiff regime [5].

The main features of the addition of the magnetic nanoparticles on the phase transition behavior of the $8 \mathrm{CB}$ liquid crystal are a decrease of both the $I-N$ and $N-S m-A$ phase transition temperatures, the narrowing of the nematic range, and the increase of the effective critical exponent $\alpha_{\text {eff }}$ describing the heat capacity anomaly of the $N-\mathrm{Sm}-A$ transition. In an effort to, at least qualitatively, understand these effects one may resort to a mean-field expansion of the free energy density $F$ in terms of the nematic order parameter $S$ and (the amplitude of) the Sm- $A$ order parameter $\psi$ and their coupling. In analogy with a mean-field description for liquid crystalline mixtures [38] and for the effects of nonmesogenic solutes $[39,40]$, where similar effects were observed, one can consider the following expansion for $F$ in terms of the order parameters $S$ and $\psi$ :

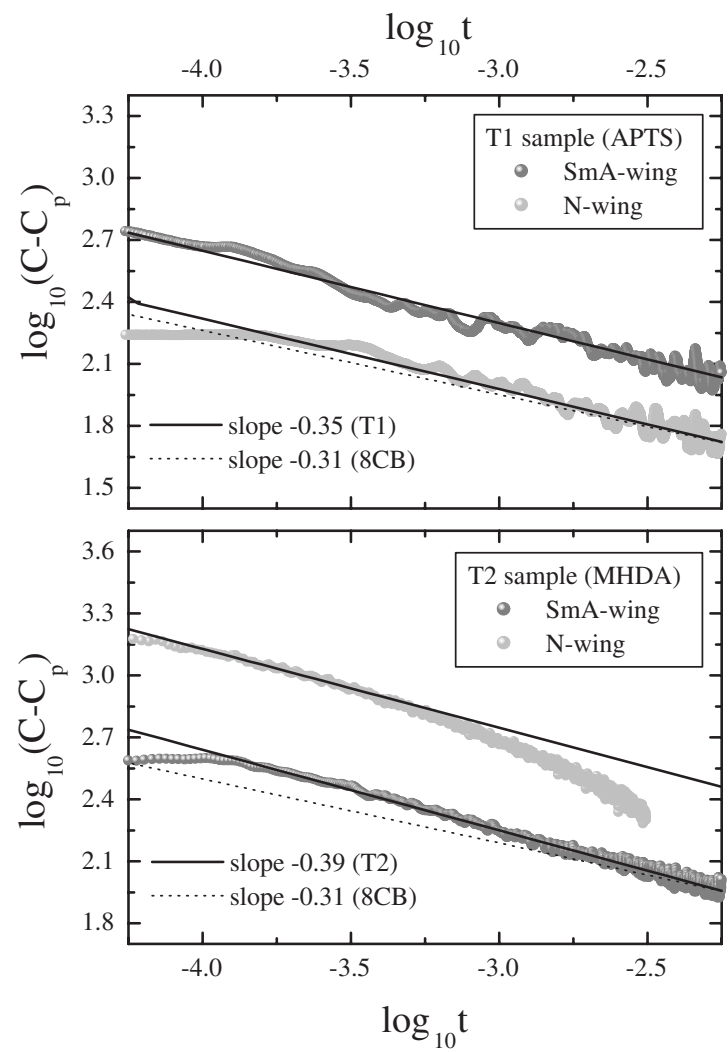

FIG. 4. The derivation of $\alpha_{\text {eff }}$ from the slope of the quantities $C-C_{p}$ and reduced temperature $t$ in a logarithmic plot, for T1 (top) and T2 (bottom). The fitting was done within the $\log _{10} t$ range of $(-4.0,-2.5)$. The points for $\log _{10} t \leqslant-4.0$ were excluded from the fit due to the rounding effects very close to $T_{c}$. Particularly for the $N$ side of the T2 sample, the data for $\log _{10} t \geqslant-3.3$ are ruled out of the fit, since the $N$ range is shrunk and the adjacency of the $I-N$ transition affects the $N$ wing of the $N-\mathrm{Sm}-A$ transition. The black solid lines represent the linear fits of the two sides $(N$ and $\mathrm{Sm}-A)$ of the transition. The dotted lines denote the slope of pure $8 \mathrm{CB}$ for comparison.

$$
\begin{aligned}
F(S, \psi)= & F_{N}(S)+\frac{1}{2} \alpha \psi^{2}+\frac{1}{4} \beta \psi^{4}-C \psi^{2} \delta S+\frac{1}{2 \chi} \delta S^{2}+A \psi^{2} x \\
& +D \psi^{2} \delta S x+\cdots,
\end{aligned}
$$

with

$$
F(S)=F_{o}(I)+\frac{1}{2} A_{0} S^{2}-\frac{1}{3} B_{0} S^{3}+C_{0} S^{4}+E S^{2} x+\cdots
$$

In Eq. (4) $F_{0}(I)$ is the free energy of the isotropic phase at $T_{N I}$, and $A_{0}=\alpha\left(T-T_{0}\right)$. The parameters $\alpha, A_{0}, B_{0}$, and $C_{0}$ are considered positive constants. In Eqs. (3) and (4) $x$ is a measure for the amount of nanoparticles added to the liquid crystal. In Eq. (3) the first five terms are identical to the Landau-de Gennes expression for the $N-\mathrm{Sm}-A$ transition of pure compounds with the coupling terms between $S$ and $\psi$ included [41]. $\alpha=\alpha_{o}\left(T-T_{c}\right)$, with $T_{c}$ the transition temperature, and $\chi(T)$ is a response function that is large near $T_{N I}$ but decreases with decreasing the temperature. In the smectic phases one has $\delta S=S-S_{o}(T)$, with $S_{o}$ the nematic order pa- 
rameter in the absence of smectic ordering. $\alpha_{0}, \beta$, and $C$ are assumed to be positive constants. The sign of the constants $A$ and $D$ in Eq. (3) as well as of the constant $E$ in Eq. (4) depend on the effect of the interactions of the nanoparticles with the liquid crystal. Rewriting in Eq. (4) the last term as $E S^{2}=E\left(\delta S+S_{0}\right)^{2} x$, minimizing Eq. (3) with respect to $\delta S$, and inserting the result in Eq. (3) gives (to the lowest order in $x$ )

$$
\begin{gathered}
F=F_{N}+\frac{1}{2} a^{*}(T) \psi^{2}-\frac{1}{4} \beta^{*} \psi^{4}, \\
T_{N I}(x)=T_{N I}(0)-\frac{2 E x}{\alpha}, \\
\alpha^{*}=\alpha+2 A x\left(1+\frac{2 C E S_{0} \chi^{*}}{A}\right), \\
\beta^{*}=\beta-2 C^{2} \chi^{*}+4 D C \chi^{*} x\left(1+4 E C \chi^{*}\right), \\
T_{N A}(x)=T_{N A}(0)-2 a_{0}^{-1} A x\left(1+4 E C \chi^{*}\right),
\end{gathered}
$$

with

$$
\chi^{*}=\frac{\chi}{1+2 E S_{0} x}, .
$$

From Eqs. (6) and (9) it follows that for decreasing $T_{N I}$ and $T_{N A}$ with increasing $x$, as observed here, both $A$ and $E$ must be positive. The sign of $\beta^{*}$ in Eq. (8) determines the order of the $N-\mathrm{Sm}-A$ phase transition. It is positive for a second-order transition, negative for a first order one, and zero at the tricritical point on the $N-\mathrm{Sm}-A$ transition line [41]. For pure 8CB $(x=0)$ Eq. (8) reduced to $\beta^{*}=\beta-2 C^{2} \chi$. The $N$-Sm- $A$ transition in pure $8 \mathrm{CB}$ is still second order, its $\alpha_{e f f}=0.31$, and its rather narrow nematic range of $7.03 \mathrm{~K}$ indicate that it is close to a tricritical point. For the colloidal mixtures T1 and T2 the observation of a larger value for $\alpha_{e f f}$ and smaller nematic ranges indicate that both are even closer to the tricritical point with still positive but smaller $\beta^{*}$ values and nanoparticles-induced coupling between the smectic and the nematic order parameters. One can thus conclude that the coupling constant $D$ is negative for both systems. The effects are, however, much larger for T2 than for T1. Since the magnetic nanoparticles are identical and the concentrations are the same, the difference results from the difference in the particles' coating.

On the bases of our macroscopic calorimetric data it is not possible to arrive at a microscopic picture about the specific interaction between the $8 \mathrm{CB}$ molecules and the two types of coating of the nanoparticles. However, from preliminary studies there seems to be some indications that in the case of APTS the orientation is parallel and perpendicular for MHDA [42]. In the latter case one can expect a larger disturbance of the liquid crystalline order. This seems to be corroborated with the large depressions of $T_{N I}$ and $T_{N A}$ for T2 (MHDA), albeit more for $T_{N I}$ than for $T_{N A}$.

\section{SUMMARY}

We have experimentally studied the impact of magnetic nanoparticles on the $I-N$ and $N-S m-A$ transitions of $8 \mathrm{CB}$, aiming to explore the until recently unresolved role of the surface coating upon the phase transition behavior. The $I-N$ transition remains weakly first order, albeit being less sharp and with a reduced amount of latent heat compared to pure $8 \mathrm{CB}$. For the $N-\mathrm{Sm}-A$ transition the results of this work show a crossover from anisotropic criticality towards tricriticality, similar to what was observed in the case of a nonmesogenic solute dispersed in the same LC compound $[39,40]$. The influence of the MHDA coating is much larger than the one of APTS and, thus, the thermodynamic behavior clearly diversifies depending on the surface coating of the nanoparticles.

\section{ACKNOWLEDGMENTS}

This research was financially supported by the Fund for Scientific Research Flanders (FWO, Project No. G. 0230.07). G.C. acknowledges the support of FWO and of Research Fund of K. U. Leuven (Project No. OT/07/035).
[1] T. Bellini, L. Radzihovsky, and N. A. Clark, Science 294, 1074 (2001), and references therein.

[2] G. S. Iannacchione, Fluid Phase Equilib. 222, 177 (2004), and references therein.

[3] T. Bellini, N. A. Clark, C. D. Muzny, L. Wu, C. W. Garland, D. W. Schaefer, and B. J. Olivier, Phys. Rev. Lett. 69, 788 (1992).

[4] L. Wu, B. Zhou, C. W. Garland, T. Bellini, and D. W. Schaefer, Phys. Rev. E 51, 2157 (1995).

[5] G. S. Iannacchione, C. W. Garland, J. T. Mang, and T. P. Rieker, Phys. Rev. E 58, 5966 (1998).

[6] M. Marinelli, A. K. Ghosh, and F. Mercuri, Phys. Rev. E 63, 061713 (2001).

[7] R. L. Leheny, S. Park, R. J. Birgeneau, J. L. Gallani, C. W. Garland, and G. S. Iannacchione, Phys. Rev. E 67, 011708
(2003).

[8] T. Jin and D. Finotello, Phys. Rev. E 69, 041704 (2004).

[9] G. Sinha, J. Leys, C. Glorieux, and J. Thoen, J. Non-Cryst. Solids 351, 2780 (2005).

[10] A. Roshi, G. S. Iannacchione, P. S. Clegg, R. J. Birgeneau, and M. E. Neubert, Phys. Rev. E 72, 051716 (2005).

[11] G. Cordoyiannis, S. Kralj, G. Nounesis, Z. Kutnjak, and S. Žumer, Phys. Rev. E 75, 021702 (2007).

[12] D. Liang and R. L. Leheny, Phys. Rev. E 75, 031705 (2007).

[13] Z. Kutnjak, S. Kralj, G. Lahajnar, and S. Žumer, Phys. Rev. E 68, 021705 (2003).

[14] S. Kralj, G. Cordoyiannis, A. Zidanšek, G. Lahajnar, H. Amenitsch, S. Žumer, and Z. Kutnjak, J. Chem. Phys. 127, 154905 (2007).

[15] S. Tripathi, C. Rosenblatt, and F. M. Aliev, Phys. Rev. Lett. 
72, 2725 (1994).

[16] S. A. Różański and J. Thoen, Liq. Cryst. 35, 195 (2008).

[17] I. Muševič, M. Škarabot, U. Tkalec, M. Ravnik, and S. Žumer, Science 313, 954 (2006).

[18] S. Kaur, S. P. Singh, A. M. Biradar, A. Choudhary, and K. Sreenivas, Appl. Phys. Lett. 91, 023120 (2007).

[19] M. Čopič, A. Mertelj, O. Buchnev, and Y. Reznikov, Phys. Rev. E 76, 011702 (2007).

[20] D. Spoliansky, V. Ponsinet, J. Ferré, and J. P. Jamet, Eur. Phys. J. E 1, 227 (2000).

[21] I. Potočová, P. Kopčanský, M. Koneracká, L. Tomčo, M. Timko, J. Jadzyn, and G. Czechowski, J. Magn. Magn. Mater. 252, 150 (2002).

[22] C. Scherer and A. M. Figueiredo Neto, Braz. J. Phys. 35, 718 (2005).

[23] F. H. Li, J. West, A. Glushchenko, C. I. Cheon, and Y. Reznikov, J. Soc. Inf. Disp. 14, 523 (2006).

[24] F. Brochard and P. G. de Gennes, J. Phys. (Paris) 31, 691 (1970).

[25] J. Rault, P. E. Cladis, and J. P. Burger, Phys. Lett. 32A, 199 (1970).

[26] L. Liébert and J. Martinet, J. Phys. (France) Lett. 40, L363 (1979).

[27] S. H. Chen and N. M. Amer, Phys. Rev. Lett. 51, 2298 (1983).

[28] P. Fabre, C. Casagrande, M. Veyssie, V. Cabuil, and R. Massart, Phys. Rev. Lett. 64, 539 (1990).
[29] L. J. Martínez-Miranda, K. Mc Carthy, L. K. Kurihara, J. J. Harry, and A. Noel, Appl. Phys. Lett. 89, 161917 (2006).

[30] L. J. Martínez-Miranda, K. Mc Carthy, L. K. Kurihara, and A. Noel, Mol. Cryst. Liq. Cryst. 435, 747 (2005).

[31] J. Thoen, H. Marynissen, and W. Van Dael, Phys. Rev. A 26, 2886 (1982).

[32] J. Thoen, Int. J. Mod. Phys. B 9, 2157 (1995).

[33] D. Davidov, C. R. Safinya, M. Kaplan, S. S. Dana, R. Schaetzing, R. J. Birgeneau, and J. D. Litster, Phys. Rev. B 19, 1657 (1979).

[34] T. C. Lubensky and J. H. Chen, Phys. Rev. B 17, 366 (1978).

[35] S. Larochelle, M. Ramazanoglu, and R. J. Birgeneau, Phys. Rev. E 73, 060702(R) (2006).

[36] H. Haga and C. W. Garland, Phys. Rev. E 56, 3044 (1997).

[37] M. Ramazanoglu, S. Larochelle, C. W. Garland, and R. J. Birgeneau, Phys. Rev. E 75, 061705 (2007).

[38] G. Nounesis, C. W. Garland, and R. Shashidhar, Phys. Rev. A 43, 1849 (1991).

[39] K. Denolf, B. Van Roie, C. Glorieux, and J. Thoen, Phys. Rev. Lett. 97, 107801 (2006).

[40] K. Denolf, G. Cordoyiannis, C. Glorieux, and J. Thoen, Phys. Rev. E 76, 051702 (2007).

[41] P. G. de Gennes and J. Prost, The Physics of Liquid Crystals (Oxford University Press, Oxford, 1993).

[42] L. J. Martínez-Miranda (unpublished). 